\title{
Estrategias de promoción de bancos comerciales a nivel de sus oficinas
}

\author{
Borgucci, Enmanuel ${ }^{*}$
}

\section{Resumen}

La promoción se convierte en una necesidad desde hace más de un lustro, cuando los bancos comerciales, ante el aumento de la competencia y sobre todo debido a la crisis bancaria del 94 , han procurado que sus gerentes de oficina dejen de ser solamente especialistas en técnicas bancarias. Cada vez mas tienen la necesidad de salir a la calle para ofertar los servicios de las instituciones que representan, en un mercado cada vez más escéptico y reducido. Aunque los gerentes se encuentran con una serie de limitaciones internas y externas a la hora de promocionar a su banco, existe una variedad de alternativas, que desde el punto de vista de la promoción pueden manejar a fin de lograr óptimos resultados en su gestión como vendedores de servicios financieros. Este trabajo expone, mediante el análisis de resultados empíricos obtenidos en el $85 \%$ de las oficinas ubicadas en Maracaibo, el conjunto de estrategias de promoción que en la actualidad aplican los gerentes de bancos comerciales, ubicados en la ciudad de Maracaibo. Además, se pretende proponer una serie de mecanismos por medio de los cuales, ésta importante función del gerente bancario puede ser llevada a cabo con éxito por el responsable de la oficina.

Palabras clave: Estrategias de promoción, bancos comerciales, oficina bancaria.

Recibido: 98-09-14. Aceptado: 99-03-26

* Profesor Agregado en la Facultad de Ciencias Económicas y Sociales de la Universidad del Zulia. Magister en Banca y Finanzas en la Universidad Rafael Urdaneta 1993 y Magister de Gerencia de Empresas Mención Mercadeo de la Universidad del Zulia 1996. Tlf: (061)-549458 y $014-9662183$. 


\section{Promotion Strategies for Commercial Banks at the Branch Office Level}

\section{Abstract}

Advertising has become a necessity over the past five years, a period in which commercial banks, faced with growing competition and above all because of the bank crisis of 94 , have required that their bank office managers be more than specialists in banking techniques. Every day the need to venture out into the street and offer institutional services is greater, in a market which is becoming smaller and more skeptical. Even when managers encounter a series of internal and external limitations in the promotion of their bank, there are a variety of alternatives that from a promotional point of view, they can manage with the objective of optimizing their results and their ability as financial service salesmen. This paper presents a series of promotion strategies which are presently being applied by commercial bank managers in the city of Maracaibo, based on an analysis of empirical results obtained in a study of $85 \%$ of Maracaibo bank offices. This study also proposes a series of mechanisms by which this most important function of bank managers can be carried out successfully.

Key words: Promotion strategies, commercial banks, bank offices.

\section{Introducción}

Los Bancos Comerciales destinan una enorme cantidad de recursos para la puesta en marcha de planes de mercadeo, que incluyen un conjunto significativo de programas y actividades destinadas a captar un mayor contingente de clientes tanto de depósitos como de créditos. Los planes de mercadeo incluyen actividades de patrocinio, programas publicitarios, apertura de nuevas oficinas; diseño, mejora o eliminación de servicios asi como la conformación de una fuerza de venta en el área corporativa destinada a captar negocios. Los planes de mercadeo bancario se caracterizan por ser documentos en donde se tratan de tomar por adelantado, de forma consciente y si es posible explicita, un conjunto de decisiones; en donde se establecen metas de desempeño y los recursos a ser empleados. En consecuencia, tratan de coordinar ordenada y sistemáticamente los ciclos de desarrollo del producto/servicio, el ciclo de ventas y el ciclo de apoyo (preventa y postventa).

No obstante, una de las debilidades que generalmente presentan esos planes de mercadeo bancarios lo constituye el hecho de que no bajan adecuadamente al punto donde el Banco Comercial contacta efectivamente al cliente. Se reconoce la importancia de la oficina bancaria dentro de la cadena de distribución, pero no se asume con facilidad el hecho 
de que es en las oficinas donde se deben elaborar y poner en marcha planes de mercadeo, los cuales deben ser liderados por su gerente. Muchas de las grandes estrategias de mercadeo desarrolladas por algunos de los principales bancos comerciales en Venezuela han fracasado precisamente por presentar graves deficiencias en la puesta en marcha de planes en elámbito de sus oficinas. Deficiencias que parten desde la carencia de investigación que proporcione información pragmática para ayudar a los ejecutivos de los bancos en su planificación estratégica (Roth y van der Velde, 1994) hasta la incapacidad de ofrecer un buen servicio como un conjunto de beneficios que tienen implícita una dimensión psicológica que supone estima, éxito y prestigio (Grubbs y Reidenbach, 1993).

Este artículo intenta exponer la situación actual de la Banca Comercial en materia de mercadeo pero desde el punto de vista de sus estrategias de promoción en el ámbito de la oficina. En tal sentido se hizo uso de los datos obtenidos en el estudio de campo desarrollado en 1995, referente a las estrategias de promoción realizadas por la banca comencial establecida en la ciudad de Maracaibo. El estudio fue desarrollado en un total de 77 oficinas de las 90 que estaban activas como producto de la crisis financiera que afectó al sistema financiero venezolano desde diciembre de 1994. La tesis central de este artículo consistirá en demostrar que la banca comercial local carece de un adecuado mecanismo de planificación de la promoción y ventas en el ámbito de sus oficinas y/o sucursales, siendo esta una actividad realizada con un alto nivel de informalidad, en donde el gerente de la oficina tiene un escaso control tanto de la planificación como de su ejecución; tanto antes de la crisis financiera del 94 como ahora, ese proceso está fuertemente mediatizado por decisiones tomadas en la oficina principal. En consecuencia, la labor del gerente de la oficina, en este contexto, es la de dirigir tareas y no de trazar horizontes acordes con su entorno particular.

\section{El mercadeo bancario en las oficinas}

El mercadeo, en el ámbito de la oficina bancaria, es un proceso que tiene como finalidad satisfacer efectivamente las demandas de servicios bancarios de los clientes que, tomando en cuenta las consideraciones o lineamientos estratégicos generados desde la oficina principal, se adaptan al entorno económico y sociocultural de la localidad donde se ubica la oficina.

Inicialmente, se consideró el enfoque de mercadeo bancario desarrollado por Badoc (1978), el cual parte de consideraciones de orden estratégico, siendo uno de los primeros en plantear este concepto al mercadeo bancario en el ámbito de la oficina y muchas de sus ideas son de enorme aplicación; pero debido a que se hace más énfasis en la dirección por objetivos, tiende a generar grandes diferencias entre los valores de las personas que laboran en la oficina y la realidad a la que se tienen que enfrentar en cada contacto con la clientela tradicional o potencial.

En consecuencia, los planteamientos de Badoc se complementarán, con varios enfoques como el de Grubbs y Reidenbach $(1993,9)$, que parten de la pers- 
pectiva de que en la banca, los servicios de alta calidad no significan 10 mismo para los banqueros que para los clientes, es decir, existe una percepción asimétrica entre banqueros y clientes. Estos autores sustentan la afirmación de que la calidad del servicio depende de que todos en el banco suministren, en cada transacción (en cada contacto cara a cara o no), un servicio de alta calidad o "satisfacer a los clientes y no solo ahorrarles molestias o inconveniencias".

Además, hay que considerar que la oficina bancaria es una cadena en el sistema de distribución total del banco, por lo que se debe partir de una definición de canal de distribución bancario. En este sentido, seguimos lo planteado por Roth y van der Velde $(1994, i v)$, quienes consideran que un Sistema de Distribución al Menudeo (SDM) se define como: "Los sistemas de suministros al menudeo incluyen, sin estar limitados por ello, a las instalaciones físicas de entrega de servicios como las sucursales y edificios, que emplean personas que están en contacto directo con los usuarios. También abarcan los canales tocnológicos, incluyendo a cajeros automáticos, puntos de venta, bancos en su casa, y otras tecnologias de autoservicio, asi como los puntos de contacto indirecto con el usuario, como el teléfono y el correo".

Por último, se estimó conveniente recurrir a Stemper $(1995, x i i)$, quien considera que el éxito de una sucursal es en gran parte responsabilidad de su director o gerente, visión que es compartida con Badoc; sin embargo, éste autor plantea el hecho de que las agencias bancarias, por lo general, actúan ante el cliente de forma pasiva, hacen en el mejor de los casos, lo que éste les dice y nunca se preocupan por intentar agregar valor a sus operaciones. Parte de que los usuarios obviamente no conocen ni todos los productos ofrecidos, ni toda la normativa operativa y tampoco todas las respuestas. Según el autor: "El valor se crea cuando el personal puede ayudar a los clientes a tomar las mejores decisiones.

Badoc (1978), considera que la primera tarea que debe tomar en cuenta un gerente de oficina, es asumir ciertas decisiones de índole estratégica que lo obligan a comprometerse personalmente. Se puede admitir cierta descentralización de las responsabilidades en la elaboración y puesta en marcha del plan en el ámbito de los funcionarios de la oficina, pero el arbitraje de las propuestas y la coordinación de la acción de promoción sólo deben ser asumidos por el gerente de la oficina. Aquí es donde entra la pregunta de: ¿Qué cosas funcionan bien y cuales no? (Stemper, 1995: 7). Es decir, desde un ambiente de diálogo, el director de la agencia debe compartir con sus funcionarios lo que se debe mejorar y lo que funciona según lo establecido. Tal vez las causas del funcionamiento deficiente se encuentren en no considerar adecuadamente acerca de hacia donde debe ir la agencia y lo que los empleados consideran que debería ser la agencia, es decir, la cultura de la agencia.

Los autores antes mencionados, de una forma u otra subrayan la necesidad de que el gerente de la oficina bancaria debe elaborar una lista de chequeo referente a las limitaciones externas e internas. Con respecto a las limitaciones externas, se deben considerar: la situación geográfica, número de comercios, núme- 
ro de empresas de servicio, nivel de desarrollo del corredor vial, el número de empresas industriales, flujo peatonal, las facilidades de acceso a las instalaciones de la oficina, condiciones de seguridad para los clientes; la presión del Estado por medio de la Política Monetaria (tasas de interés, operaciones de compra y ventas de Títulos de Estabilización Monetaria, etc.), la reglamentación de los diferentes órganos de la administración pública nacional, estadal (regional o municipal, los niveles de competencia interbancaria, etc. Entre los factores de orden interno se pueden mencionar: las limitaciones ejercidas por la oficina principal del instituto bancario, la gama de servicios que se ofrecen, la infraestructura de servicios existente, las políticas de tasas de interés aplicadas, las políticas implementadas para el cobro de comisiones, el reclutamiento del personal, las estrategias de incentivación y de adiestramiento diseñadas, etc.

En consecuencia, las limitaciones de un gerente de oficina, no solamente son variadas, sino también abundantes, algunas veces simultáneas y en muchos casos conflictivas. Sin embargo, puede, si tiene el interés, el conocimiento y la función del mercadeo, jugar con los diferentes factores, algunos de los cuales son: participar activamente en la formulación de los objetivos de la oficina en el ámbito de gerencia o vicepresidencia regional; organizar, con pocos recursos, las relaciones institucionales sabiendo vender las ideas a la oficina principal; administrar el personal a su cargo ocupándose de su inducción, adiestramiento, su mejoramiento profesional (comunicando expe- riencias y conocimientos); llevar el control de las actividades que se desarrollan e impliquen un mejoramiento de la posición competitiva de la oficina; administrar la prospección de nueva clientela, organizando visitas a clientes importantes de la zona; seleccionar las estrategias de telemercadeo, correo directo y ventas personales más apropiadas a las posibilidades de la agencia; administrar la información, controlando constantemente el grado de obsolescencia de los diferentes archivos de los clientes; por último, puede jugar con la distribución de los servicios bancarios dentro de las metas establecidas por la oficina principal.

Es también indispensable realizar un análisis del entorno, dado que la oficina no está aislada del resto de las empresas y comercios que la circundan. El gerente de la oficina debe pronosticar, al más largo plazo posible, las tendencias que pueden caracterizar ese entorno. Este pronóstico debe basarse en los siguientes elementos:

1. Evolución comercial del corredor vial, recabando información acerca del desarrollo cuantitativo y/o cualitativo de la utilización del suelo urbano.

2. Datos demográficos de la zona, los cuales son necesarios para el conocimiento de la clientela actual y potencial. Permite conocer si la oficina ha cubierto su zona de influencia. Lo anterior puede ser obtenido de los organismos oficiales del ramo, de las Cámaras de Industriales o de Comercio local o por medio de un trabajo de campo propio de la agencia.

3. En cuanto a la competencia, lo primero que no debe hacer un gerente de oficina es asumir una actitud de auto- 
suficiencia y menosprecio del competidor. En su lugar, lo debe conocer lo más profundamente posible. Entre los datos que pueden contribuir al conocimiento de la competencia se encuentran: En qué servicios compiten, tipo de clientela, su localización, imagen del competidor (su prestigio, solvencia, experiencia), grado de penetración en el mercado local, análisis de los servicios ofrecidos por la competencia, Las tasas pasivas y activas cobradas, los argumentos utilizados por la competencia para vender sus servicios, determinar los medios de marketing y sobre todo las estrategias de promoción en el punto de venta, las estrategias de telemercadeo, de relaciones institucionales y de correo directo utilizadas por la competencia.

Lo anterior derivará en una matriz de Definición de Oportunidades (Stemper, 1995: 47), las cuales deben ser cuidadosamente identificadas, debido a que lo que aparentemente es una oportunidad, a lo mejor no lo es en absoluto, o lo es en forma limitada.

Se debe tomar en cuenta además de lo anterior, el conocimiento de la clientela actual que posee la oficina. Este conocimiento no debe estar referido solamente a un número de cuenta; debe ser un elemento clave para la evaluación del mercadeo en el ámbito de la oficina, incluyendo la rentabilidad de los distintos tipos de clientela en términos de coste-beneficio y desembocar en una verdadera gestión por segmentos de clientela. Pero además, es necesario que el valor esti- mado por el consumidor acerca de la capacidad de los servicios ofrecidos por la oficina para satisfacer sus necesidades, sea el máximo (Kotler, 1996: 8). Ahora bien, es comün que el sistema de información en el banco sea centralizado, por lo que el gerente debe presionar y buscar que la oficina principal tome en cuenta sus necesidades de información. La obtención de la información comercial del cliente es tarea de todos los integrantes de la oficina. Cada miembro del personal debe ser formado, por medio de cursos de capacitación, acerca de la obtención de información fidedigna y confiable de los clientes, construcción de base de datos, análisis de la información, etc.

El proceso de análisis estratégico interno y externo de la oficina culmina con una evaluación de servicios (Grubbs y Reidenbach, 1993:123). La razón de la auditoría consiste en que para hacer estrategias de promoción a nivel de la oficina es necesario establecer indicadores para medir la forma y calidad de las interacciones del personal con el cliente en las actividades de ventas directas, Telemercadeo, Correo Directo, Promoción en el lugar de Ventas y Relaciones Institucionales. La evaluación buscará comparar lo que piensan los funcionarios y empleados acerca de su desempeño e investigar la percepción acerca de la labor de los funcionarios y empleados pero desde el punto de vista del cliente (mediante muestreo en los días de mayor actividad). Los aspectos a ser evaluados serían los siguientes:

- La preocupación del banco por el cliente. 
- El respeto del banco para con el cliente.

- Agilidad en dar respuestas a dudas, objeciones y problemas en los servicios prestados.

- Capacidad del banco para convencer al cliente de las bondades del servicio ofrecido.

- Capacidades y habilidades del personal de ventas para explicar los servicios ofrecidos.

- Calidad de las respuestas para atender dudas, problemas y objeciones del cliente.

- Facilidad para contactar la persona adecuada para atender problemas de los clientes.

- Tiempo que debe esperar el cliente para ser atendido.

- Amabilidad en el trato para con los clientes.

- Apariencia personal de los empleados que atienden a los clientes.

- Tratamiento que recibe el cliente cuando la oficina está congestionada.

Los resultados de la evaluación pueden presentar coincidencias o discrepancias entre la oficina y el cliente. Las coincidencias pueden referirse a aspectos positivos o negativos de la gestión. $A$ la oficina le conviene tener una percepción positiva del cliente en la totalidad de los aspectos evaluados. En el caso de las discrepancias, la oficina debe prestar atención cuando los clientes perciban una gestión negativa en comparación con la percepción que sobre los mismos aspectos evaluados tenga el personal y hasta el mismo gerente.

Es necesaria la realización de reuniones periódicas en donde el gerente, junto con todos los empleados de la ofici- na busquen las causas de las discrepancias entre lo que percibe el cliente y la visión que los empleados tienen de su actuación. Esto con la finalidad de generar el estado de ánimo necesario que permita la búsqueda consensual y se planteen las estrategias a seguir para corregir las desviaciones que en un momento dado se presenten.

Otro elemento que debe ser considerado por el gerente una vez cumplidos los pasos previamente esbozados, es el establecimiento del pronóstico de la oficina. Este pronóstico permite conocer cómo será la oficina dentro de cierto número de años si el gerente mantiene las mismas directrices que se han mantenido hasta ahora, sobre todo en materia de promoción (Badoc, 1978:215). Cuando el pronóstico es desfavorable, el gerente, en el peor de los casos, debe hacer una reconversión de sus estrategias de promoción para atacar las nuevas realidades.

Ahora bien, tanto si el pronóstico es favorable o no, el gerente de la oficina debe implementar una serie de medidas tendientes a mejorar su posición competitiva. Lo anterior se resume en: formulación de objetivos y metas y establecimiento de las decisiones y acciones a implementar.

La primera labor consiste en definir los parámetros para medir su desempeño. Naturalmente, la fijación de las metas está condicionada por lo que establezca la Dirección General del Banco Comercial. Pero la oficina tiene que transformar los datos recogidos de la clientela y tomar en cuenta el análisis interno y externo. Los objetivos deben ser distribuidos en el transcurso del año, pero más aún, exigir a la oficina principal la información acerca 
de las campañas de los nuevos servicios a lanzar. Ahora bien, una forma para establecer objetivos consiste en determinarlos por servicios y tipo de clientelas; en lo referente a los tipos de servicios, se pueden establecer a partir de aquellos servicios que deben ser apoyados, vigilados o lanzados. Desde el punto de vista de la fijación de metas, se establecerian por: clientelas que se deben crear, las que se deben mantener, las que se deben desarrollar y las que se deben eliminar.

Por último, antes de someter a la oficina a un seguimiento de todo lo que se ha planeado, es necesario poner en orden un Plan de Utilidades, ya que: "Los bancos necesitan planes de utilidades $y$ las sucursales tambiérn". (Stamper, 1995: 80). En el ámbito de la oficina es necesario hacer congruentes los ingresos recibidos por las operaciones de crédito y conexas con los gastos de administración y financieros.

\section{Las estrategias de promoción en la oficina bancaria}

Cuando se realizó el estudio de las estrategias utilizadas por la banca en el ámbito de sus oficinas, se consideró conveniente estudiar tres de las múltiples estrategias que se podian adoptar: ventas directas, promoción en el lugar de ventas y telemercadeo. Las tres constituyen el núcleo por medio del cual se puede desa. rrollar una efectiva captación y colocación de recursos desde los clientes de depósitos hacia los clientes de crédito en sus diferentes modalidades.
La venta directa es la base para que toda oficina bancaria comercial pueda realizar una gestión exitosa de captación y colocación de recursos. Se consideraron como elementos claves para la gestión de ventas: la fuente de datos usados por la oficina; las formas de selección de los clientes; el lugar de contacto de la clientela; el número de vendedores; los cursos realizados por el personal de ventas; los incentivos otorgados al personal de ventas; el tipo de metas trazadas y las formas de costear las actividades de ventas directas.

Según los resultados de la encuesta, para ofrecer servicios especiales, 64 oficinas, es decir el $83 \%$ de las oficinas encuestadas, utilizaron listados generados por las computadoras de las direcciones centrales, acerca de los clientes de cuentas corrientes y de ahorro. En segundo lugar, el $74 \%$ de los casos utilizaron listados de crédito. En último lugar, un $60 \%$ hizo uso de los sistemas de información en línea.

Lo anterior señala que los bancos dependen, en gran medida, de información obtenida con un retraso de entre 1 y 3 días, lo cual hace laborioso el trabajo de actualizar los registros internos necesarios para organizar las actividades de venta. No obstante, los bancos están modernizando sus sistemas de información y se observa una mayor presencia de sistemas en línea.

Por lo general, las oficinas cruzan los listados de cuentas de depósitos con los de crédito para derivar clientes potenciales para crédito; sin embargo, los criterios adoptados para la selección no se 
complementan con la información que existe, por ejemplo, en los ficheros de los clientes, que constituye una fuente importante de detección de características sociodemográficas de la clientela.

Más bien, se apela a las referencias dadas por el gerente, los funcionarios o empleados que contactan al cliente cada vez que éste visita la oficina.

Algo interesante de resaltar, es el hecho de que 2 gerentes hicieron la captación de sus clientes saliendo de la oficina, realizando visitas exploratorias; un gerente respondió que se valió de revistas especializadas tanto del sector industrial, petrolero o comercial y otro gerente se valió del ejecutivo de cuenta de la banca corporativa para captar clientes. Estos resultados se confirman cuando el $71 \%$ de las actividades de ventas directas son realizadas dentro de la oficina.

Lo anterior demuestra, en primer lugar, que gran parte de la actividad promocional del gerente está dentro de la oficina, quizás porque existen oficinas con pocos funcionarios autorizados para atender la masa de documentos generados por las operaciones de captación o colocación. Por otro lado, el personal que pudiera estar dedicado a las actividades de ventas, tiene que apoyar las actividades operativas de caja, de prueba o de crédito. En síntesis, existe la necesidad de una reorganización de funciones, tareas y procedimientos que estén adaptados a la tecnología existente o a la nueva tecnología que se implemente en la oficina.

El gerente de la oficina, en base a su experiencia como banquero y siguiendo las políticas emitidas por la Dirección General del Banco Comercial, establece una serie de criterios para seleccionar aquellos clientes que podrian generar un volumen importante de negocios y sobre todo de ingresos. Entre los criterios más importantes para la selección de clientela, se encuentran: los saldos compensatorios $(79 \%)$ y la segmentación según la experiencia de crédito $(70 \%)$. Como se puede notar, la banca sigue utilizando criterios clásicos en materia de selección de clientes. El primero mide, por un lado la lealtad del cliente para con la institución frente a la competencia y por el otro lado, expresa, en términos del banco captador, la capacidad de potencial de pago, en el caso de un cliente de crédito o el nivel de ingresos si es solo un cliente de depósitos. La experiencia de crédito, mide el historial de pago del cliente y la capacidad de acceder a nuevos servicios.

El número de personas destinadas a las actividades de ventas directas tuvo una media de 5 empleados, pero con una desviación también de 5 empleados. Este resultado muestra de que así como existen oficinas con 5 o más empleados trabajando en labores de ventas, en otras su presencia es nula, quizás debido a que se dedican a las actividades operativas. En consecuencia, existe una discontinuidad en la realización de esta función debido a que solo se hace énfasis en las ventas cuando la oficina no cumple sus metas de captación o colocación.

En cuanto a la formación del personal de ventas por parte de la institución bancaria, es importante destacar que en el $58 \%$ de las mismas no se realizaron cursos de ventas directas y sólo el $35 \%$ de las oficinas encuestadas realizaron entre uno y cuatro cursos al año.

Lo anterior demuestra que la labor de ventas se realiza en la mayoría de los 
casos, por personas que si bien pudieran dominar técnicas básicas no acceden a las modernas técnicas de ventas de servicios financieros. Otro aspecto a resaltar es que las oficinas envian pocos empleados a realizar cursos, generalmente empleados nuevos del departamento de crédito, la secretaria del gerente o algún empleado de atención al público.

Lo anterior es más grave si se considera que, el $32 \%$ de las oficinas encuestadas manifestaron no poseer ningún programa de incentivos para sus vendedores, debido a que son actividades realizadas por el gerente, el cual cuenta con bonos por metas cumplidas más que por ventas y negocios captados exclusivamente. El $29 \%$ de las oficinas otorgan un bono fijo por metas cumplidas, pero esta vez a los empleados en conjunto el cual se divide entre el número de empleados que tenga la oficina. Solo el $10 \%$ de las oficinas encuestadas otorgan una comisión variable por montos captados según el tipo de servicio ofrecido.

Como se puede notar, lejos de diseñarse incentivos específicos por cuentas o clientes captados, los incentivos de ventas se diluyen dentro de los demás incentivos creados. Es así como los empleados dedicados a las actividades de venta, excepto el gerente y los funcionarios, prestan poco interés a la venta de los servicios de la institución. Esta falta de interés se manifiesta en el desconocimiento de los detalles tanto operativos como jurídicos de aquellos servicios más importantes o nuevos que el banco puede ofrecer, así como en el trato que se le brinda al cliente.

Los objetivos que se trazan las oficinas en materia de captación y coloca- ción de recursos son variados y cada vez más se inscriben dentro del resto de las metas de carácter operativo que tiene que cumplir la oficina como canal de distribución. El $27 \%$ de las oficinas diseñan sus objetivos considerando el número de cuentas y al mismo tiempo el monto a captar y colocar en dinero mensualmente, pero sin especificar en qué tipo de cuentas en particular se debe poner énfasis. Por otro lado, el $13 \%$ de las oficinas prefieren establecer sus metas por número de cuentas a ser captadas y su monto mensual, especificando el tipo de servicio a ser vendido. Otras oficinas establecen metas más largas, considerando los períodos de cierre contable de las instituciones financieras, que en Venezuela es cada seis meses. El $11 \%$ de las oficinas establecen sus metas semestrales por número de cuentas y montos captados sin especificar el tipo de cuenta y otro $11 \%$ to hacen especificando el tipo de cuenta a captar. Por último, el $38 \%$ de las oficinas consultadas no establecen metas de ventas.

Como se podrá apreciar, se enfatiza el total de cuentas a captar en términos genéricos en lugar de las metas según los segmentos o servicios. Se captan clientes nuevos, soiamente cuando el gerente observa que en sus registros (listados o sistema en línea), la mayoría de sus clientes están lo suficientemente cruzados o saturados de servicios del banco junto a una mayor brecha entre lo que se debe captar y colocar y lo realmente captado y colocado por esa oficina en el período de evaluación.

Por otro lado, se establecen metas específicas al lanzar un servicio nuevo, cuando un servicio tradicional del banco 
ha caído apreciablemente tanto en número de cuentas como en monto en dinero, 0 en la oportunidad en que se le exige a la oficina la captación de un determinado número de clientes para las empresas filiales (cuando el banco no es universal).

Por último, la oficina depende para gastos de promoción, en el $79 \%$ de los casos de los aportes que les otorga la dirección general, mientras que el $17 \%$ de las oficinas consultadas se valieron de sus recursos propios, sobre todo de los ahorros internos de partidas asignadas para gastos y la iniciativa del personal en aportar dinero propio y/o de los premios por metas y concursos ganados. Un ejemplo, es la práctica consistente en que se carga, por orden del gerente de la oficina, la cuenta de gastos para Publicidad y Relaciones con el Público y/o la cuenta de gastos auxiliares Anuncios, Viajes y Agasajos, Donaciones, Liberalidades y Otros, abonando las cantidades asignadas a las actividades de promoción que se han acordado realizar.

La segunda herramienta de promoción considerada fue la promoción en el lugar de ventas. En la oficina bancaria, es importante tomar en cuenta las herramientas utilizadas en el punto de venta y el costo de su implementación. La herramienta de promoción en el lugar de venta más utilizada $(76 \%)$ fue el uso de material impreso (dípticos, trípticos o folletería) y en segundo lugar la demostración al cliente de los beneficios de los servicios ofrecidos por el banco. En menor escala, un $23 \%$ de las oficinas consultadas, hicieron uso de espacios dentro de la oficina para exhibiciones y demostraciones (por ejemplo, el uso del cajero interactivo) y un $22 \%$ utilizaron láminas acrílicas.
Es importante destacar, en primer lugar, que la utilización del material impreso depende de los suministros que envie la dirección general. Por otro lado, el mismo solo se reproduce para que el público tenga información general acerca del servicio ofertado más no se utiliza para complementar la venta directa, dado que no está diseñado para demostrar, dar pruebas o aclarar objeciones acerca de las bondades, deficiencias aparentes 0 reales de los servicios ofrecidos. Finalmente, se reproducen con motivo del lanzamiento o relanzamiento de servicios aumentados (Kotler, 432:1996), es decir, servicios con atributos nuevos y diferenciados respectos a los competidores.

Por otro lado, este tipo de actividades, como las de ventas directas, depende principalmente de las asignaciones de la dirección general. El $63 \%$ de las oficinas consultadas expresaron que el material impreso viene de la dirección general previamente diseñado y elaborado, es decir, que el gasto se realiza a ese nivel y la oficina es sólo un canal de divulgación de la información. En consecuencia, la promoción de ventas en la oficina sigue una actitud pasiva por parte del personal y su gerente, quienes solo esperan aleatoriamente que el cliente se acerque para informarle de los servicios del banco y comenzar, eventualmente, un proceso de venta.

El Telemercadeo constituye la tercera herramienta de promoción que complementa la venta directa. Dentro de las oficinas de los bancos comerciales, el telemercadeo, que incluye el uso del fax, puede ser utilizado para la venta de servicios o como medio para realizar actividades de postventa. No se consideró aquí el telemercadeo por medio del nú- 
mero 800 de tarifa gratis para el cliente o las actividades de promoción que se pueden hacer por medio del internet (que hacen uso también de las líneas telefónicas), sino las iniciativas que parten del gerente de la oficina, que utilizando un recurso disponible en su oficina, le permitan captar clientes, ya sea por medio de un telemercadeo de salida (llamadas desde la oficina) como de entrada (llamadas hacia la oficina).

Ahora bien, para abordar el telemercadeo desde la oficina de un banco comercial, se consideraron los siguientes elementos: El tipo de aplicaciones que la oficina le otorga al telemercadeo, la fuente de datos, las formas de seleccionar la clientela, el número de operadores y los cursos realizados; la clase de incentivos recibidos, dado que ellos también laboran como vendedores y; los tipos de objetivos establecidos.

Se observó que el telemercadeo es poco utilizado, ya que poco menos del $20 \%$ de las oficinas encuestadas lo utilizan a la hora de lanzar un nuevo servicio del banco, para aumentar la captación de recursos y para conocer las quejas de los clientes. Es de resaltar que si bien estos porcentajes son bajos, las razones para esta situación se encuentran en la insuficiencia de líneas telefónicas en la oficina y a su vez en la oferta restringida de nuevas lineas analógicas o digitales por parte de la empresa responsable del servicio telefónico a nivel nacional. Muchas de las oficinas consultadas cuentan con algo menos de cinco líneas telefónicas y las lineas disponibles se utilizan para las acti- vidades operativas tanto en el área de depósitos como en el área de crédito.

Entre las fuentes de datos para realizar las actividades de telemercadeo por parte de la oficina bancaria se encuentran el registro electrónico de clientes $(27 \%)$, seguido por los listados de depósitos (25\%) y los listados de los clientes de créditos (23\%).

Es de destacar, que es en el telemercadeo donde los gerentes hacen más uso del registro electrónico de clientes, aunque esta proporción sigue siendo baja. El resto de las oficinas que tienen una idea de lo que es el telemercadeo o que lo pueden implementar, ya sea por iniciativa propia o impuesta por la dirección general, siguen haciendo uso de los listados de clientes de cuenta corrientes, de ahorro o de los listados de pagarés, descuentos, créditos en cuentas corrientes o líneas de créditos.

Con la información anterior, las oficinas estructuran la lista de clientes para contactarlos telefónicamente por medio de una segmentación de carácter económico-financiero; clasifican sus clientes tanto por su experiencia de crédito como por su capacidad de pago. De igual forma, los saldos compensatorios son otro criterio utilizado. En resumen, en las oficinas bancarias privan los criterios anteriores y se busca que los cumplan todos. Una vez cubiertos los requisitos, se busca analizar el tipo de cliente, esto es, la solvencia moral o las visitas que realiza a la oficina, ya que eso es señal de que mantiene vínculos con el banco pero por medio de otros negocios. 
En las operaciones de telemercadeo participan un promedio de 4 empleados con una desviación también de 4 empleados, con 2 cursos en promedio al año. Es de hacer notar que ésta cantidad de personas no se emplean constantemente en el telemercadeo de la oficina. En realidad, la oficina utiliza personal adicional cuando existe una campaña de lanzamiento de un servicio nuevo o para relanzar un servicio existente. Mas bien, el teléfono se emplea como medio para cobrar a clientes de crédito, llamar a clientes que tienen sobregiro en cuenta corriente o cuando el gerente está interesado en conversar con un cliente que considera interesante a la hora de hacer negocios para el banco.

Debido a la naturaleza discontinua de la actividad de telemercadeo en la oficina, los incentivos son casi inexistentes. De hecho el $77 \%$ de las oficinas no otorgan ningún tipo de incentivo. Este sólo se otorga como parte de un incentivo global por metas cumplidas durante el mes o el semestre según la estructura de metas del banco comercial a nivel nacional. Unicamente el $5 \%$ de las oficinas otorgan un incentivo de tipo variable por montos captados, lo cual es un ejemplo de la poca importancia que se le otorga a esta importante y útil herramienta de promoción.

Al analizar los objetivos establecidos para el telemercadeo, se aprecia el escaso valor que se le concede a la promoción vía telefónica. En efecto, el $77 \%$ de las oficinas consultadas no establecen ningún tipo de metas para esta actividad, quizás por las limitaciones mencionadas de infraestructura comunicacional. Las metas se fijan en función de lo que se haga por medio de la venta directa, refle- jando que el telemercadeo no es en sí una función sino un instrumento representado por un teléfono o un fax que facilita, en ciertas ocasiones, las operaciones antes y después de realizar la venta.

\section{Conclusiones}

En materia de promoción la banca de capital regional y nacional así como la de capital extranjero presente en esta región carece de un adecuado mecanismo de planificación de la promoción y ventas en el ámbito de sus oficinas, siendo ésta una actividad realizada con un alto nivel de informalidad por el gerente de la oficina $y$ sus funcionarios; donde el gerente de la oficina tiene un escaso control tanto de la planificación, como de la ejecución de los programas y actividades concretas derivadas de ella. Más bien se observó, que tanto antes de la crisis financiera del 94 y con más fuerza hoy en día, ese proceso está fuertemente mediatizado por decisiones tomadas en la oficina principal.

Lo anterior se evidencia por el hecho de que las ventas directas, aunque contando con sistemas de información en proceso de modernización o de automatización, no logran agrupar de forma rápida y efectiva a las personas o empresas que le pudieran interesar a la oficina.

Por otro lado, el número de personas dedicadas a las actividades de ventas directas, de promoción de ventas y telemercadeo es insuficiente, a pesar de que se han notado esfuerzos por automatizar los procesos de back-office, lo que muestra que la modernización no está seguida por la existencia de procesos más expeditos para realizar transacciones. En necesario que todo el personal sea entre- 
nado en el arte de la venta de servicios bancarios y financieros en sus diferentes modalidades, no solamente para captar clientes, sino para captar los mejores y mantenerlos.

Lo anterior se acompaña de una deficiente política de incentivos; los que existen lo que hacen es potenciar la desmotivación del personal entrenado y asignado a las actividades de promoción. Esa política de incentivos se sustenta en la inexistencia de metas especificas y un presupuesto cada vez más limitado por las directrices de la oficina principal. En consecuencia, lejos de servir como medio de comunicación productiva tanto para el cliente como para el banco, la oficina bancaria está más cerca de ser solo un simple canal suministrador de servicios bancarios de carácter tradicional (pago de cheques, recepción de depósitos, otorgar pagarés, etc.).

En consecuencia, el gerente de una oficina bancaria así como los de división o vicepresidentes ejecutivos de una entidad bancaria deben en primer lugar, estar atentos a la evolución de un entorno bancario signado por la inestabilidad, la presencia de la banca extranjera y por la presencia de un nuevo tipo de cliente que está sacando lecciones de la crisis financiera del 94.

Por otro lado, los gerentes de oficina deben identificar los procesos críticos comunicativos tanto detrás del mostrador como enfrente del mostrador de la oficina. No siempre lo que comunican los empleados acerca de lo bien que lo hacen es suficiente, se necesita siempre la opinión del cliente.

Además, se necesita formar equipos de trabajo para diagramar los procesos actuales en la mezcla comunicacional de la oficina, definir las unidades de medición y modificar aquellos procesos críticos que atenten contra el éxito de la gestión de la oficina.

Por último, lo anterior implica que tanto el gerente como los empleados de la oficina deben abordar estos retos con espíritu de entusiasmo realista, vigilando constantemente el desempeño establecido en la planificación con los resultados de la gestión, tanto a nivel interno como a nivel del público que asiste a la oficina.

\section{Bibliografía citada}

Badoc Michel. (1978). El Marketing en los Bancos Europeos. España. Editorial Tecniban, S.A.

Grubbs. M Ray y Reidenbach R. Eric. (1993). "Banca de Excelencia". Cómo lograr servicios de alta calidad en los bancos. México. Editorial Panorama, S.A. Primera edición en Español.

Kotler Philip. (1996). "Dirección de Mercadotecnia. Análisis, Planificación, Implementación y Contror. México. Pretince Hall Hispanoamericana, S.A.

Roth Aleda V. y Van der Velde Marjolijn. (1994). "Estrategias para la banca de menudeo". Grupo Editorial Iberoamérica. México.

Stemper R.G. (1995). "Administración exitosa de sucursales bancarias". Grupo Editorial Iberoamérica. México. 